\title{
Assessing Corticospinal Tract Asymmetry in Unilateral Polymicrogyria
}

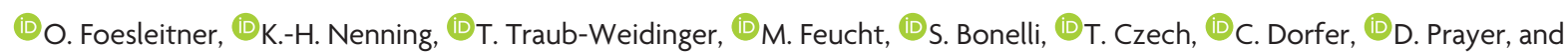
(D) Kasprian

\section{ABSTRACT}

BACKGROUND AND PURPOSE: Asymmetry of the corticospinal tract in congenital lesions is a good prognostic marker for preserved motor function after hemispherectomy. This study aimed to assess this marker and provide a clinically feasible approach in selected cases of unilateral polymicrogyria.

MATERIALS AND METHODS: Corticospinal tract asymmetry of 9 patients with unilateral polymicrogyria substantially affecting the central region was retrospectively assessed on axial TIWI and DTI. Volumes of the brain stem and thalamus and DTI parameters of the internal capsule were measured. Two neuroradiologists independently rated the right-left asymmetry at 4 levels along the corticospinal tract. DTI tractography was used to determine the motor cortex within polymicrogyria, with task-based functional MR imaging available in $3 / 9$ cases.

RESULTS: Visual assessment of the brain stem asymmetry showed excellent correlation with quantitative measures on both TIWI and color-coded DTI maps $(P=.007$ and $P=.023)$. Interrater reliability regarding structural and DTI-based corticospinal tract asymmetry was best at the midbrain (Cohen $\kappa=0.77, P=.018$ ). Three patients underwent functional hemispherectomy with postsurgical stable motor function, all showing marked corticospinal tract asymmetry preoperatively. Following the DTI-based corticospinal tract trajectories allowed identifying the presumed primary motor region within the dysplastic cortex in $9 / 9$ patients, confirmed by functional MR imaging in $3 / 3$ cases.

CONCLUSIONS: Visual assessment of corticospinal tract asymmetry in unilateral polymicrogyria involving the motor cortex is most reliable with TIWI and color-coded DTI maps at the level of the midbrain. Pronounced asymmetry predicts preserved motor function after hemispherectomy. DTI-based tractography can be used as a guidance tool to the motor cortex within polymicrogyria.

ABBREVIATIONS: $A D=$ axial diffusivity; $C S T=$ corticospinal tract; $F A=$ fractional anisotropy; $P M G=$ polymicrogyria; $R D=$ radial diffusivity

P olymicrogyria (PMG) is a cortical malformation due to abnormal postmigrational development, which is morphologically characterized by an excess of small gyri. ${ }^{1}$ Depending on the localization and the extent of cortical involvement, clinical man-

Received February 18, 2018; accepted May 2.

From the Departments of Biomedical Imaging and Image-Guided Therapy (O.F., K.-H.N., T.T.-W., D.P., G.K.), Pediatrics and Adolescent Medicine (M.F.), Neurology (S.B.), and Neurosurgery (T.C., C.D.), Medical University of Vienna, Vienna, Austria. Paper previously presented as a poster at: Annual Meeting of the European Congress of Radiology, March 1-5, 2017, Vienna, Austria; Annual Meeting of the German and Austrian Societies of Neuroradiology, October 5-8, 2016, Cologne/Germany; and Annual Meeting of the European Conference of Epileptology, September 11-15, 2016, Prague, Czech Republic.

Please address correspondence to Gregor Kasprian, MD, Department of Biomedical Imaging and Image-Guided Therapy, Medical University of Vienna, Waehringer Guertel 18-20, 1090 Vienna, Austria: e-mail: gregor.kasprian@meduniwien.ac.at

三 Indicates article with supplemental on-line table

Indicates article with supplemental on-line photos.

http://dx.doi.org/10.3174/ajnr.A5715 ifestations range from selective impairment of higher cognitive functions to severe epilepsy with intractable seizures. Epilepsy surgery is a delicate matter in these patients. Complete resection of the epileptogenic zone is a strong prognostic factor for surgical success $^{2}$ and supports extensive surgery. However, there is often preserved function in the polymicrogyric cortex, ${ }^{3-5}$ with the risk of postoperative de novo deficits.

Küpper et $\mathrm{al}^{6}$ recently formulated "rules" for the prediction of preserved grasping ability after hemispherectomy, stating that "marked asymmetry of the brain stem and, more specifically, marked asymmetry of the corticospinal tract (CST) within the brain stem, predict preservation of grasping ability in patients who can grasp preoperatively." This conclusion is based on observations in a large but heterogeneous cohort of different pre-, peri-, and postnatally acquired lesions not specifically focusing on PMG.

Due to the extreme divergence from normal anatomy, charac- 
teristic landmarks for the identification of the central sulcus cannot be used in PMG. Thus, structural imaging alone fails to localize the preserved motor function within the dysplastic cortex. Shown to be concordant with intraoperative cortical mapping, , $^{3,7}$ fMRI serves as a noninvasive criterion standard for localizing the motor area. Because PMG is typically symptomatic in early childhood, ${ }^{8}$ the results of preoperative task-based fMRI studies in these young uncooperative patients are frequently vague and often degraded by severe motion artifacts.

By measuring the anisotropic motion of water, diffusion tensor imaging can provide imaging correlates of the fiber architecture of the human brain. ${ }^{9}$ Tractography visualizes these fiber tracts by combining voxel-based anisotropy values, constructing continuous fiber bundles. The accuracy and reliability of DTIbased tractography in the visualization of the corticospinal tract has been confirmed by intraoperative correlation, mainly in brain tumor surgery. ${ }^{10}$ To the best of our knowledge, the use of DTI as a neuroradiologic tool to localize preserved motor function within PMG has not been investigated.

This study had the following objectives: 1) propose a clinically feasible imaging approach to assess CST asymmetry in unilateral polymicrogyria, 2) check diffusion-based tractography as guidance to the presumed motor area within the dysplastic cortex, and 3) investigate whether the "rule" of CST asymmetry as a good prognostic factor for postsurgical motor function preservation specifically applies to cases of unilateral PMG involving the central region.

\section{MATERIALS AND METHODS}

The retrospective data analysis was approved by the Ethics Committee of the Medical University of Vienna (1139/2012).

Inclusion criteria for this single-center retrospective study were existing presurgical MR imaging examinations performed between 2008 and 2017 with dedicated epilepsy imaging protocols, including a T1-weighted isovoxel sequence $\left(1-\mathrm{mm}^{3}\right.$ voxel size) and a DTI sequence (16 or 32 gradient encoding directions, b-values of 0 and $700 \mathrm{~s} / \mathrm{mm}^{2}, 2$ - to 3 -mm slice thickness) from $3 \mathrm{~T}$ (patients 1-3) or 1.5T scanners (patients 4-9). All patients were diagnosed with PMG substantially ( $>$ two-thirds of the motor cortex) involving the primary motor cortex of 1 hemisphere. $\mathrm{Pa}-$ tients with a malformation in the contralateral hemisphere or any other structural alteration along the CST or a prior neurosurgical procedure were excluded.

In a retrospective chart review, 9 cases of unilateral PMG involving the central region (6 right-hemispheric) were identified (for anatomic images see On-line Fig 1). The median age at MR imaging was 7 years (range, 3-34 years). Patient characteristics are outlined in the On-line Table.

Brain stem volume was obtained by manual segmentation of the left and right halves on axial T1WI from below the mammillary bodies to the obex as anatomic landmarks to ensure intersubject comparability using ITK-SNAP (www.itksnap.org; On-line Fig 3). ${ }^{11}$ We determined the midline considering the central canal, the anterior median fissure, and the posterior median sulcus. Anatomic correctness was further checked by visual inspection of the segmented volume in the sagittal and coronal planes and as $3 \mathrm{D}$ volume. The ratio of contralesional-to-ipsilesional brain stem volumes quantifying asymmetry was then calculated (brain stem volume ratio):

$$
\frac{(\text { Contralesional }- \text { Ipsilesional Volume })}{(\text { Contralesional }+ \text { Ipsilesional Volume })}
$$

Diffusion tensor images were quantitatively analyzed by manually drawing ROIs on axial planes (DSIStudio; Johns Hopkins University, Baltimore, Maryland). Diffusion parameters of the posterior limb (being the normal anatomic location of the CST) and of the whole internal capsule were measured. Fractional anisotropy (FA), ADC, axial diffusivity (AD), radial diffusivity (RD) of the full axial area of the brain stem, and the normal anatomic location of the CST only were obtained from 1 axial slice below the mammillary bodies (On-line Fig 2). A DTI-based CST asymmetry ratio was then calculated with a score of zero indicating symmetry:

$$
\frac{(\text { Contralesional }- \text { Ipsilesional Value })}{(\text { Contralesional + Ipsilesional Value })}
$$

Two experienced academic neuroradiologists (G.K. and D.P., with 5 and 30 years of experience) blinded to clinical data independently assessed the asymmetry of the CST on 4 levels (internal capsule, cerebral crus, medulla, and spine) and the thalamus. Asymmetry was rated as 0 (no asymmetry), 1 (mild), 2 (moderate), or 3 (severe) and summed into an asymmetry index ranging from 0 to 15 . A positive and high asymmetry index indicated a pronounced asymmetry and a smaller CST ipsilateral to the lesional hemisphere. Next to the structural images, the readers were shown the color-coded diffusion tensor images on the level of the midbrain (1 slice below the mammillary bodies, corresponding to the quantitative measurement). The simultaneous assessment of structural and diffusion tensor images aimed to reflect the true clinical setting.

Task-based fMRI data acquired at $3 \mathrm{~T}$ were available in 3 patients $(1.8 \times 1.8 \times 4.0 \mathrm{~mm}$ slice thickness, $128 \times 128$ matrix, $\mathrm{TE}=35 \mathrm{~ms}$, TR $=3000 \mathrm{~ms}$, flip angle $\left.=90^{\circ}\right)$. The motor task consisted of alternating blocks of activation (finger tapping, ankle flexion and extension) and visual stimulation, each lasting $30 \mathrm{sec}-$ onds with a total time of 5 minutes. Due to severe paresis, patient 1 could only move both hands simultaneously. fMRI data were preprocessed on SPM12 software (http://www.fil.ion.ucl.ac.uk/ $\mathrm{spm} /$ software/spm12). Functional images were coregistered with the original T1WI, realigned and smoothed with a Gaussian kernel of 8 -mm full width at half maximum, and motion-corrected with standard parameters.

Deterministic tractography of the CST was performed by manually drawing ROIs on axial slices into the internal capsule and lateral to the lateral ventricle (DTIStudio). In patients in whom functional imaging was available, fiber tracking was performed first and then compared with the fMRI activation map by measuring the Euclidean distance from the center of the blood oxygen level-dependent cluster to the presumed motor area indicated by DTI.

The results of clinical neurologic examinations were retrieved from the medical charts. An experienced neurologist or neuropediatrician systematically performed all examinations. Hand motor strength was graded according to the Medical Research Coun- 

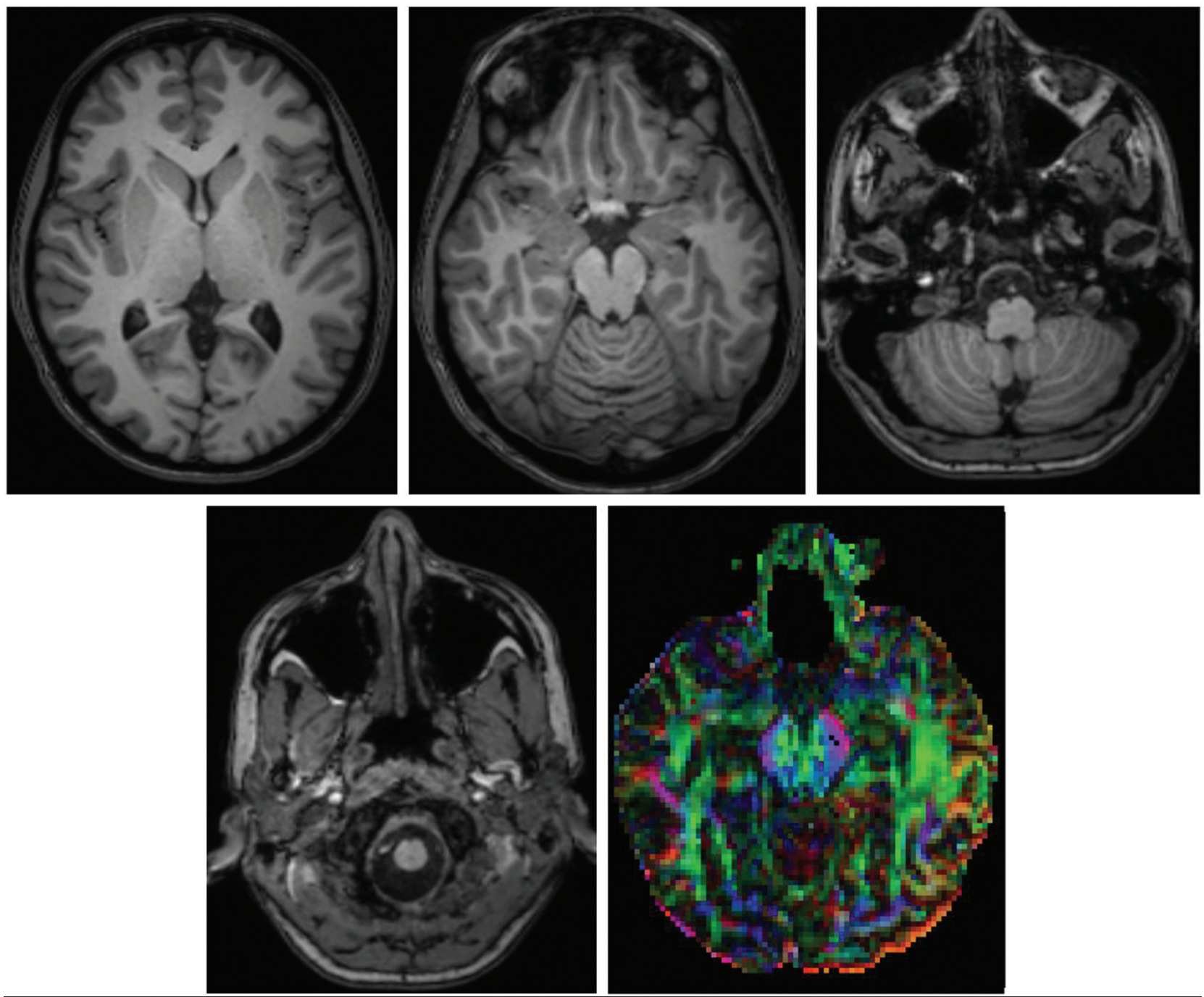

FIG 1. CST characterization in patient 3: TIWI at different levels of the corticospinal tract (internal capsule, cerebral crus, medulla, spine), color-coded FA color map (brain stem). Asymmetry was altogether rated as no/mild asymmetry.

cil scale ${ }^{12}$ and, together with fine motor skills, was categorized as "minimal" or "distinct" impairment.

Imaging and clinical values were correlated with bivariate analyses and the Pearson coefficient (SPSS, Version 22.0; IBM, Armonk, New York), while the interrater reliability was calculated using the Cohen coefficient. The threshold of significance was set at $<.05$.

\section{RESULTS}

Figures 1 and 2 show structural and functional imaging in patient 3. Another example of structural and functional imaging for patient 1 can be found in On-line Figs 4 and 5.

\section{Structural Asymmetry}

The mean left-right asymmetry ratio of the manually segmented brain stem was $0.1 \pm 0.05$ (range, 0.03-0.17). The mean left-right asymmetry ratio of the anterior parts of the brain stem (as the normal location of the CST) was $0.14 \pm 0.06$ (range, 0.07-.22).

The visual assessment of the CST at 4 different levels plus the thalamus resulted in a median asymmetry index of 9 (range, 1-12) for 1 rater and 7 (range, 3-11) for the second rater.
Interrater reliability in the assessment of CST asymmetry was most robust at the level of the cerebral crus. Excellent congruence was reached by categorizing the asymmetry degree into no/minimal asymmetry or moderate/severe asymmetry (Cohen $\kappa=1.0$, $P=.003)$. The other levels of assessment did not result in significant agreement (Cohen $\kappa=0.21-0.6 ; P>.05)$.

Visual assessment of the brain stem asymmetry showed an excellent correlation with manual brain stem volumetry $(P=$ $.007)$.

Clinical hand motor impairment correlated with the visual assessment of the structural CST asymmetry at the internal capsule $(P=.001)$, cerebral crus $(P=.014)$, and the overall structural asymmetry index $(P=.002)$. The structural CST asymmetry assessed at the level of the thalamus, medulla, and spine did not correlate significantly with the clinical hand motor function $(P>.05)$.

\section{Diffusion Tensor-Based Asymmetry}

The mean ratio of FA values in the internal capsule was $0.05 \pm 0.05$ (range, $0.0-0.16$ ). The mean ratio of FA values in only the posterior limb of the internal capsule was $0.08 \pm 0.05$ (range, 0.02-0.18). 

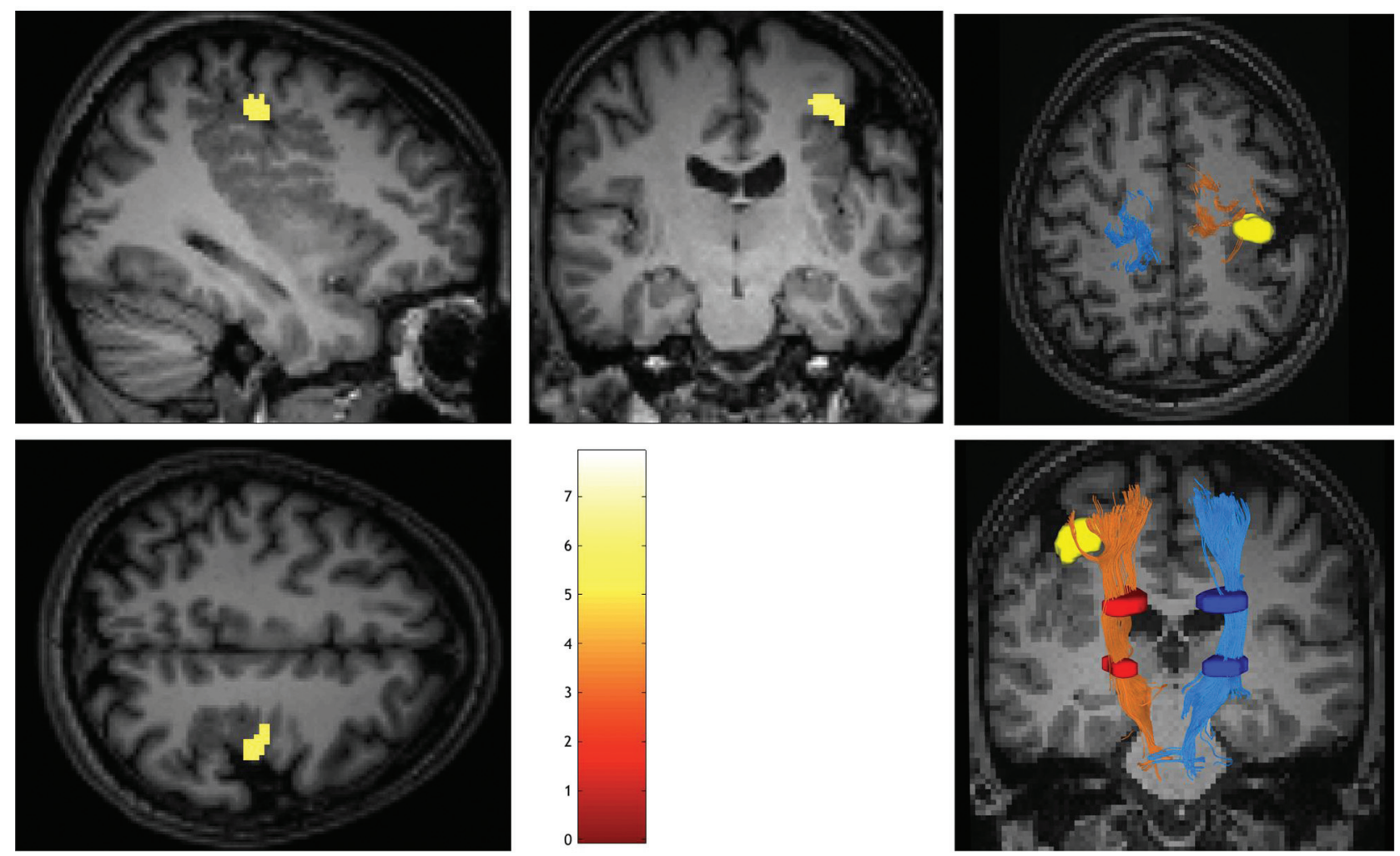

FIG 2. fMRI activation map (hand motor task, family-wise error of $<.05$, 6-voxel minimum cluster size) and DTI tractography of the CST in patient 3.

Mean ratios of DTI parameters in the brain stem taken from 1 axial slice (below the mammillary bodies) were as follows (Online Fig 2): mean FA $=0.05 \pm 0.06$ (range, $-0.01-0.2$ ), mean $\mathrm{ADC}=-0.02 \pm 0.07$ (range, $-0.15-0.06)$, mean $\mathrm{AD}=0.01 \pm$ 0.03 (range, $-0.04-0.6$ ), mean $\mathrm{RD}=-0.05 \pm 0.09$ (range, $-0.21-0.05)$. Mean ratios of diffusion tensor parameters in only the anterior half of the brain stem measured at 1 axial slice were as follows: mean $\mathrm{FA}=0.01 \pm 0.09$ (range, $0.0-0.26$ ); mean $\mathrm{ADC}=$ $-0.09 \pm 0.06$ (range, $-0.17-0.01$ ); mean $\mathrm{AD}=-0.03 \pm 0.07$ (range, $-0.11-0.1$ ); and mean $\mathrm{RD}=-0.16 \pm 0.07$ (range, -0.25 to -0.07$)$.

Diffusion tensor parameters measured at the anterior part of the brain stem correlated well with the visually assessed diffusion asymmetry at the same height $(P=.023)$. Diffusion measures from the whole manually segmented brain stem did not correlate with the raters' asymmetry index.

Visual rating of color-coded DTI maps of the brain stem resulted in a mean asymmetry score of $2 \pm 0.87$ (range, $1-3$ ) in 1 rater and $1.9 \pm 1.3$ (range, $0-3$ ) in the second rater. By using a simplified asymmetry rating of only 2 instead of 4 categories (no/minimal or moderate/severe asymmetry) an excellent interrater reliability could be achieved (Cohen $\kappa=1.0, P=.003$ ) as opposed to no significant interrater reliability using all 4 categories $(P>.05)$.

The visual asymmetry rating of the color-coded FA diffusion tensor maps at the level of the brain stem correlated with hand motor function $(P=.018)$. Quantitative diffusion tensor measures did not correlate significantly with clinical hand motor function.

\section{Motor Cortex}

Task-based functional imaging showed highly significant taskrelated activation within the polymicrogyric cortex in $3 / 3$ cases $(P<.05$, family-wise error correction; minimum cluster size, 6 voxels). Patients were 19, 26, and 34 years of age at the fMRI scan.

Tractography successfully determined the corticospinal tract bilaterally in $9 / 9$ patients and led to the primary motor cortex with a mean distance of $9 \mathrm{~mm}$ to the fMRI motor activation in $3 / 3$ cases (range, 0-17.9 $\mathrm{mm}$ ).

\section{Clinical Motor Function}

Two patients showed minimal hand function impairment (full muscle strength, minimal impairment of fine motor skills), while 7/9 patients had moderate-to-severe motor function deficits.

Three patients underwent functional hemispherectomy. Preoperatively, hand motor strength was rated Medical Research Council grade 4 in all 3 cases, with moderate-to-severe deficits in fine-motor skills in the contralateral extremity. Ipsilateral hand motor function was graded as normal. After the operation, these 3 patients were seizure-free (2-year follow-up) with no de novo motor deficits. On presurgical imaging, all 3 patients showed marked asymmetry of the CST on visual inspection (anatomic and/or diffusion tensor images) and in asymmetry quantification.

\section{DISCUSSION}

This series of unilateral polymicrogyria affecting the central region further supports the good prognostic value of CST asymmetry for postoperative motor function preservation. We showed that asymmetry of the CST can be reliably assessed with visual 
inspection of T1WI and diffusion tensor images, showing the most robust assessment at the level of the cerebral crus using a dichotomous asymmetry scale (no/minimal or moderate/severe asymmetry). Quantitative measures of the CST using brain stem volumetry and diffusion tensor parameters (FA, ADC, AD, RD) significantly correlated with the visual inspection-based asymmetry assessment. Additionally, this study proposed tractography as a guidance tool to the preserved motor area not identifiable on structural images due to the extreme divergence from normal anatomy. Proving the concept of abnormal axonal wiring in brain malformations, ${ }^{13}$ presurgical structural and tractographic visualization of the asymmetric CST indicated significantly altered structural motor connectivity in the motor cortex involving PMG.

Küpper et $\mathrm{al}^{6}$ recently defined "rules" for the prediction of postoperative hand motor function after hemispherectomy in a large cohort of pre-, peri-, and postnatally acquired lesions using manual brain stem segmentation, probabilistic tractography, and visual inspection of axial FA color maps by 1 reader. They found that in patients with preoperatively normal hand function and in patients with postnatally acquired lesions or progressive diseases, "complete loss of grasping ability must be expected." Similarly, CST asymmetry was shown to be a good prognostic factor for preserved motor function after hemidisconnection. In their cohort, they included 16 cases of PMG not explicitly located in the central region, with T1WI being available in 15 and DTI performed in 6 cases. Our series of a highly selective population of unilateral PMG involving the central region confirms these "rules." Furthermore, our study adds a clinically feasible imaging approach in the preoperative setting by showing the reliability of visual inspection by experienced neuroradiologists as opposed to also reliable but demanding quantitative analyses not practical in clinical routine (manual brain stem volumetry, DTI parameters).

Three of our patients underwent functional hemispherectomy with postoperative seizure freedom and no deterioration of motor function. This result proves the concept of potential motor function preservation in prenatally acquired lesions due to reorganization. ${ }^{6}$ Both our raters, independently and blinded to clinical data, assessed the CST asymmetry as moderate/severe in these 3 patients. This could have served as helpful prognostic information in the preoperative setting. Early-onset childhood epilepsy is often medically refractory and results in widespread brain dysfunction. ${ }^{14}$ An epilepsy operation in patients with PMG has recently been shown to be significantly better than medical treatment alone. ${ }^{15}$ Mühlebner et al ${ }^{14}$ found 5/7 patients with PMG seizure-free after a disconnective operation at our center and extended surgery to be a positive predictive marker for seizure-freedom. In adequately selected patients, an operation can achieve seizure freedom in $67 \%$ of cases. ${ }^{15}$

However, with possible function preserved within the to-beresected or disconnected brain tissue, the risk of de novo deficits must be weighed against the benefits of potential postoperative seizure freedom. Despite the associated substantial derangement of the affected cortical anatomy and the severely disrupted neuronal layering, PMG is known to have functionally relevant sensorimotor and visual representations. ${ }^{16}$ This observation is welldocumented in smaller series ${ }^{4,5,17,18}$ by fMRI, transcranial magnetic stimulation, ${ }^{5}$ and magnetoencephalography. ${ }^{19}$
PMG can potentially affect any supratentorial brain region except for the striatum, hippocampus, and gyrus rectus. ${ }^{20}$ It is more commonly found bilaterally, most frequently involving the posterior aspect of the Sylvian fissure and the frontal or parietal lobes. Although summarized as 1 pathology, the molecular and developmental etiology of PMG can vary substantially. Generally, typical unilateral PMG is today seen as a result of abnormal postmigrational development and therefore a developmental disorder that occurs relatively late in corticogenesis. ${ }^{1}$ Thus, preserved function is rather common in PMG compared with other malformations of cortical development, which lead to abnormal corticogenesis at much earlier time points and consequently more frequently cause functional cortical reorganization. ${ }^{3}$ This is confirmed by our series in which structural and diffusion tensorbased asymmetry of the CST was closely correlated with hand motor impairment.

Most interesting, unilateral PMG typically does not macroscopically affect the subcortical and deep white matter. ${ }^{20}$ This finding is in line with that in our series using deterministic tractography as a robust measure of grossly preserved sensorimotor structural connectivity in the region of PMG. Thus, DTI tractography can potentially help to identify preserved motor function within the dysplastic motor cortex, giving additional information in the setting of preoperative evaluation. Functional MR imaging is often not feasible in these pediatric and intellectually disabled patients or is only feasible using passive movements in sedation. ${ }^{21}$

In pre- or perinatal cortical lesions, preservation of ipsilateral corticospinal projections is associated with favorable functional outcome. ${ }^{22}$ Neurophysiologic studies suggest that neurons in a damaged sensorimotor cortex fail to maintain their contralateral spinal terminations, while projections from the healthy contralesional primary motor cortex increase. ${ }^{22}$ Asymmetry of the CST in early acquired lesions (ie, pre- or perinatal) is thus a good prognostic factor because it indicates structural and functional reorganization into the ipsilateral sensorimotor cortex.

\section{Limitations}

The observations of this study are based on a retrospective analysis of a relatively small population. Given the strict inclusion criteria (unilateral PMG involving the central region) and the rarity of the disease, however, this is the largest series addressing the defined aims so far. We deliberately wanted to focus on unilateral PMG affecting the central region because this has not specifically been the focus before but is of high clinical relevance in specialized centers. This study intended to elaborate an imaging approach robust and feasible for clinical routine in the preoperative work-up of patients with PMG, thus concentrating on the radiologic perspective. Only 3 patients underwent an epilepsy operation, but all had marked asymmetry of the CST preoperatively and did not show clinical de novo deficits postoperatively. Given the limited statistical power, evaluating the prognostic value of structural and tensor-based asymmetry of the CST on postoperative motor function is beyond the scope of this study, but it has been convincingly shown in a large population of pre- or perinatal lesions. ${ }^{6}$ 


\section{CONCLUSIONS}

Visual assessment of structural and diffusion tensor images of the corticospinal tract (especially at the cerebral crus) is a reliable and clinically feasible imaging approach in the preoperative work-up of patients with unilateral PMG affecting the central region. In noncompliant patients, DTI-based tractography is a useful alternative to task-based fMRI and helps in the anatomic localization of the primary motor cortex. Moreover, this study confirmed the assumption of CST asymmetry as a good prognostic factor for postoperative hand motor function in this highly selected population. Finally, it adds to the concept of preserved ipsilateral corticospinal connectivity as a plasticity mechanism in early unilateral hemispheric brain lesions.

\section{ACKNOWLEDGMENTS}

The authors thank Dr Gudrun Groeppel for her participation in the data acquisition.

Disclosures: Gregor Kasprian—UNRELATED: Consulting Fee or Honorarium: Shire, Comments: honorarium for a lecture held on November 2017; Payment for Lectures Including Service on Speakers Bureaus: Shire, Comments: honorarium for a lecture on neuroimaging in Fabry disease; Travel/Accommodations/Meeting Expenses Unrelated to Activities Listed: Philips Healthcare Europe, Comments: flight to Amsterdam for a Pediatric User Meeting in Eindhoven, Holland, February 2018.

\section{REFERENCES}

1. Barkovich AJ, Guerrini R, Kuzniecky RI, et al. A developmental and genetic classification for malformations of cortical development: update 2012. Brain 2012;135:1348-69 CrossRef Medline

2. Hader WJ, Mackay M, Otsubo H, et al. Cortical dysplastic lesions in children with intractable epilepsy: role of complete resection. J Neurosurg 2004;100:110-17 Medline

3. Janszky J, Ebner A, Kruse B, et al. Functional organization of the brain with malformations of cortical development. Ann Neurol 2003;53:759-67 CrossRef Medline

4. Nikolova S, Bartha R, Parrent AG, et al. Functional MRI of neuronal activation in epilepsy patients with malformations of cortical development. Epilepsy Res 2015;116:1-7 CrossRef Medline

5. Zsoter A, Pieper T, Kudernatsch M, et al. Predicting hand function after hemispherotomy: TMS versus fMRI in hemispheric polymicrogyria. Epilepsia 2012;53:e98-101 CrossRef Medline

6. Küpper H, Kudernatsch M, Pieper T, et al. Predicting hand function after hemidisconnection. Brain 2016;139:2456-68 CrossRef Medline

7. Yetkin FZ, Mueller WM, Morris GL, et al. Functional MR activation correlated with intraoperative cortical mapping. AJNR Am J Neuroradiol 1997;18:1311-15 Medline
8. Caraballo RH, Cersósimo RO, Fortini PS, et al. Congenital hemiparesis, unilateral polymicrogyria and epilepsy with or without status epilepticus during sleep: a study of 66 patients with long-term follow-up. Epileptic Disord 2013;15:417-27 CrossRef Medline

9. Le Bihan D, Mangin JF, Poupon C, et al. Diffusion tensor imaging: concepts and applications. J Magn Reson Imaging 2001;13:534-46 CrossRef Medline

10. Stadlbauer A, Buchfelder M, Salomonowitz E, et al. Fiber density mapping of gliomas: histopathologic evaluation of a diffusion-tensor imaging data processing method. Radiology 2010;257:846-53 CrossRef Medline

11. Yushkevich PA, Piven J, Hazlett HC, et al. User-guided 3D active contour segmentation of anatomical structures: significantly improved efficiency and reliability. Neuroimage 2006;31: 1116-28 CrossRef Medline

12. John J. Grading of muscle power: comparison of MRC and analogue scales by physiotherapists: Medical Research Council. Int J Rehabil Res 1984;7:173-81 CrossRef Medline

13. Besseling RM, Jansen JF, de Louw AJ, et al. Abnormal profiles of local functional connectivity proximal to focal cortical dysplasias. PLOS One 2016;11:e0166022 CrossRef Medline

14. Mühlebner A, Gröppel G, Dressler A, et al. Epilepsy surgery in children and adolescents with malformations of cortical development: outcome and impact of the new ILAE classification on focal cortical dysplasia. Epilepsy Res 2014;108:1652-61 CrossRef Medline

15. Cossu M, Pelliccia V, Gozzo F, et al. Surgical treatment of polymicrogyria-related epilepsy. Epilepsia 2016;57:2001-10 CrossRef Medline

16. Barkovich AJ, Gressens P, Evrard P. Formation, maturation, and disorders of brain neocortex. AJNR Am J Neuroradiol 1992;13: 423-46 Medline

17. Burneo JG, Bartha R, Gati J, et al. 4-T fMRI of the motor and sensory cortices in patients with polymicrogyria and epilepsy. Clin Neurol Neurosurg 2014;122:29-33 CrossRef Medline

18. Araujo D, de Araujo DB, Pontes-Neto OM, et al. Language and motor FMRI activation in polymicrogyric cortex. Epilepsia 2006;47: 589-92 CrossRef Medline

19. Burneo JG, Kuzniecky RI, Bebin M, et al. Cortical reorganization in malformations of cortical development: a magnetoencephalographic study. Neurology 2004;63:1818-24 CrossRef Medline

20. Barkovich AJ. Current concepts of polymicrogyria. Neuroradiology 2010;52:479-87 CrossRef Medline

21. Ogg RJ, Laningham FH, Clarke D, et al. Passive range of motion functional magnetic resonance imaging localizing sensorimotor cortex in sedated children. J Neurosurg Pediatr 2009;4:317-22 CrossRef Medline

22. Eyre JA. Corticospinal tract development and its plasticity after perinatal injury. Neurosci Biobehav Rev 2007;31:1136-49 CrossRef Medline 